\title{
¿CÓMO VUELAN LOS COHETES?
}

José Luis Comparán Elizondo

Universidad Autónoma de Nuevo León-México

Recibido: 13 - mayo - 2015, aprobado: 10 - julio - 2015

\section{Resumen}

La Universidad Autónoma de Nuevo León (México) ha alcanzado fama continental y mundial por sus logros en divulgación científica. Como una muestra y en forma exclusiva, presentamos el ensayo del Dr. José Luis Comparán Elizondo sobre el vuelo de los cohetes, dirigido a escolares, colegiales y público en general. Es un orgullo para Anales de la Universidad Central del Ecuador, mostrar un ensayo que, sin lugar a duda, estimulará la creación de trabajos similares en otras áreas y ciencias.

Palabras clave: divulgación científica, cohetes, propulsión, gases, combustión.

\begin{abstract}
The Autonomous University of Nuevo León (México) has reached continental and worldwide fame for its achievements in science. As a sample, and exclusively, we present the essay of Dr. José Luis Comparán Elizondo on the flight of rockets, designed for school and college students and for the general public. It is a pride for Annals of the Central University of Ecuador, showing an essay, that for sure, will estimulate the creation of similar works in other areas and sciences.
\end{abstract}

Keywords: popular science, rockets, propulsion, gas, combustion. 


\section{Antecedentes}

Cuando sueltas un globo lleno de aire presurizado, el aire del interior del globo está a una presión mayor que la presión atmosférica del exterior, esta diferencia de presiones, hace que el aire salga del interior del globo. Véase la Figura 1.

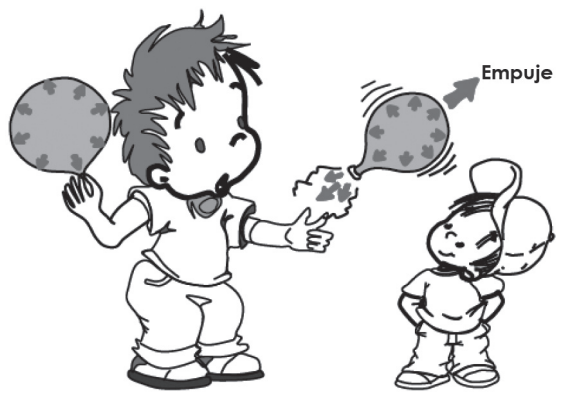

Figura 1. Cómo vuela un globo.

El globo se mueve, por el efecto del aire que deja el globo. Dicho efecto se llama "empuje". El empuje tiene dirección contraria a la del aire que abandona el globo.

En la naturaleza existen cierto grupo de moluscos marinos llamados cefalópodos, que parecen consistir de una cabeza y un montón de pies o tentáculos que les sirven para desplazarse y para sujetar sus presas; entre ellos se encuentran calamares, pulpos, sepias y nautilos. La característica principal de este grupo es que todos sus miembros salen directamente de la cabeza. Su nombre deriva del griego, kephale igual cabeza, y pous o podos, igual a pie, por lo que el nombre de estos invertebrados significa «cabeza con pies». El equivalente humano sería que nuestros brazos y piernas salieran de alrededor de nuestro cuello.

Algunos de ellos pertenecen al orden de los octópodos, es decir, tiene 8 pies o tentáculos (el pulpo), otros al orden de los decápodos, ya que tienen 10 pies o tentáculos (el calamar). Véase la Figura 2.
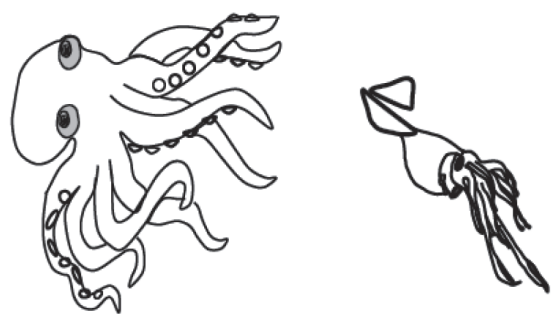

Figura 2. Izquierda octópodo llamado pulpo. Derecha decápodo de nombre calamar.

La sangre de los cefalópodos es de color azul, debido a que usan el cobre para transportar el oxígeno en lugar de hierro como lo hacemos los humanos.

\section{LA PROPULSIÓN A CHORRO}

Una de las características más importantes es que los cefalópodos poseen un sifón, este es un tubo musculoso que utilizan para expulsar agua a presión, dando lugar al empuje, permitiéndoles avanzar rápidamente usando la llamada propulsión 
a chorro, lo hacen para escapar de sus depredadores (véase la Figura 3), o bien para expulsar un chorro de tinta y enturbiar el agua, despistando a sus atacantes.

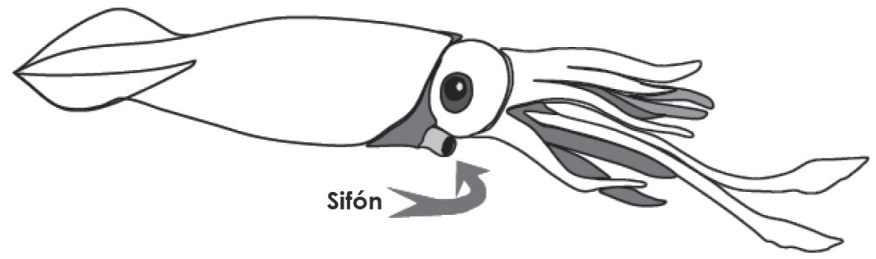

Figura 3. Dibujo de calamar donde se muestra el sifón.

El mecanismo que el calamar y el pulpo usan para producir el empuje se describe a continuación, tomando como ejemplo al calamar (véase la Figura 4).
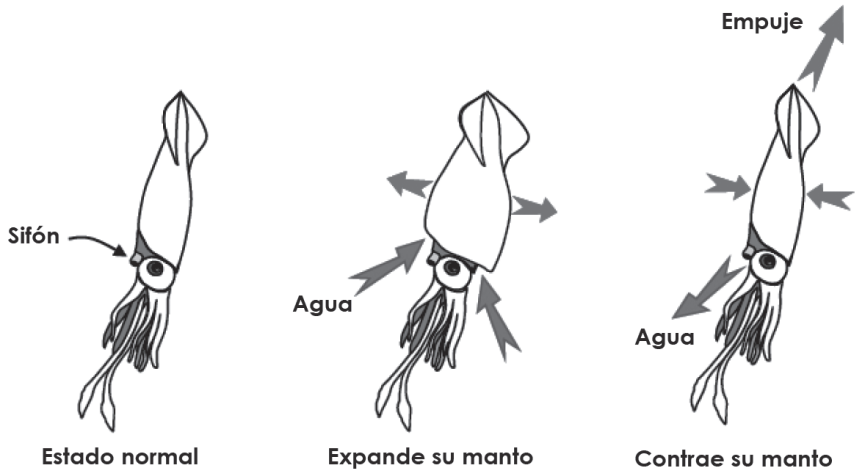

Figura 4. El calamar generando el empuje.

Paso uno, el calamar dilata su manto para introducir agua a su cuerpo.

Paso dos, el calamar contrae su manto para expulsar violentamente el agua de su cuerpo a través de su sifón, dando lugar a la propulsión a chorro, es decir, produce el empuje que hace que éste se mueva rápidamente en la dirección contraria a la del chorro de agua emitido por él.

Los cohetes vuelan de una manera similar a los ejemplos mostrados en las figuras 1 y 4. En la Figura 5, se muestra un dibujo de un cohete en movimiento, la fuerza de empuje se genera por las partículas de la combustión expulsadas a gran velocidad por la tobera del cohete.

Como a grandes alturas no hay oxígeno, para que en el cohete se realice la combustión, es necesario que el cohete mismo lleve consigo su propio oxígeno.

En los cohetes de combustible sólido, el oxígeno va mezclado con el combustible (recordar la pólvora de los cohetes de los juegos pirotécnicos). Una vez que se prende, sigue así hasta que se agota por completo.

El cohete de combustible líquido, lleva en su interior un tanque de combustible y su respectivo tanque de oxidante (oxígeno). El combustible y el oxígeno se inyectan a la cámara de combustión de los motores, al mezclarse constituyen una sustancia muy explosiva, que al encenderse producen gases de alta temperatura, éstos salen expulsados por la tobera pero al pasar por la garganta de esta, 


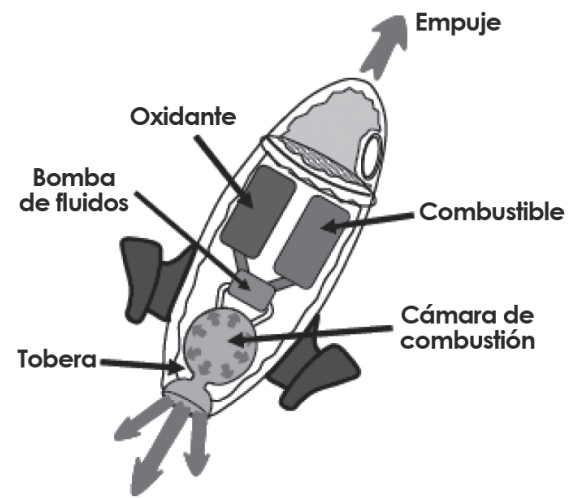

Figura 5. Cohete moviéndose a causa de la expulsión de gases de combustión a altas velocida-

des. Es la llamada propulsión a chorro. incrementan aún más su velocidad, dando lugar al empuje del cohete que lo hace moverse en dirección contraria al chorro de partículas expulsadas en forma de gas. La ventaja de este tipo de cohetes estriba en que las válvulas del combustible y oxidante se pueden cerrar ya sea manualmente o a control remoto e interrumpir la combustión y de hecho el empuje. Esto no quiere decir que al interrumpir el empuje el cohete se detenga, la nave seguirá moviéndose por inercia, dicho movimiento cumple con lo establecido en la primera ley del movimiento de Newton.

La tobera del motor del cohete, es la parte por donde se expulsa el gas de a muy alta presión y temperatura. La tobera tiene una parte estrecha (garganta) que sirve para aumentar la velocidad del gas expulsado.

Obviamente, el lector tendrá una pregunta más.

\section{¿Cómo explica la ciencia este fenómeno de propulsión a chorro?}

Le corresponde a la Física explicar este fenómeno, más precisamente a la rama de la Física llamada mecánica.

En esta última ciencia hay un principio físico que al expresarlo en lenguaje simple se puede enunciar de la siguiente manera: si tenemos un cuerpo (o sistema de cuerpos) aislado ${ }^{1}$, entonces, si a causa de fuerzas internas una parte de ese cuerpo o sistema es expulsada en una determinada dirección, el resto del cuerpo debe moverse en dirección contraria.

En lenguaje científico, debemos decir, que el principio mencionado en el párrafo anterior se llama, Principio de la conservación del momento lineal y establece lo siguiente:

Principio de la conservación del momento lineal

En todo cuerpo o sistema de cuerpos aislados, su mo-

mento lineal total, se conserva, es decir, permanece constante con el transcurso del tiempo.

El momento lineal de un cuerpo es una magnitud física vectorial (por lo que posee módulo y dirección), esta magnitud física depende de la masa $\mathrm{m}$ del cuerpo y del módulo $\mathrm{v}$ de la velocidad $\overrightarrow{\mathrm{v}}$ del mismo, se le designa por $\overrightarrow{\mathrm{p}}$ y es igual al producto de la masa del cuerpo por su velocidad, es decir $\vec{p}=m \vec{v}$.

El momento lineal nos indica que tan fácil o que tan difícil es cambiar el estado de movimiento de un cuerpo o sistema de cuerpos. Si $\overrightarrow{\mathrm{p}}$ tiene un valor pequeño,

1 Cuerpo aislado: un cuerpo (o sistema de cuerpos) aislado, es aquel que no interactúa con cualquier otro cuerpo, o mejor dicho, es un cuerpo (o sistema de cuerpos) en el que la resultante de todas las fuerzas que surgen de la interacción con otros cuerpos, se anulan entre sí unas con otras. 
se requiere una fuerza pequeña para cambiar su estado de movimiento, por el contrario, si $\overrightarrow{\mathrm{p}}$ es muy grande, se requerirá de una gran fuerza para sacarlo del reposo o para acelerarlo si está en movimiento.

Por otra parte, el empuje de los cohetes, de los pulpos y de los calamares aquí tratados, se explica de acuerdo con el principio de la conservación del momento lineal.

Como los cohetes vuelan debido al principio de conservación del momento lineal, hay que conocer con más detalle, en que consiste este principio.

\section{El principio de la conservación del Momento Lineal}

En todo sistema aislado constituido por " $\mathrm{n}$ " cuerpos, su momento lineal total:

$$
\vec{p}=\sum_{i=1}^{i=n} \vec{p}_{i}=\vec{p}_{1}+\vec{p}_{2}+\vec{p}_{3}+\ldots \quad \ldots+\vec{p}_{n}
$$

Se conserva, es decir, ¡No cambia con el tiempo!

Aplicando este principio físico al vuelo del cohete, decimos que el sistema lo constituyen dos cuerpos: uno de esos cuerpos es el conjunto de todas las partículas resultantes de la combustión que son expulsadas por la tobera del cohete, y el otro cuerpo, "al que llamaremos el resto del cohete y lo designaremos por Rcohete", lo constituyen todos los elementos del cohete incluyendo el combustible y el oxidante cuya masa disminuirá al irse quemando a razón constante en los motores del cohete.

\section{¿Cómo es la expresión matemática de este principio físico?}

Matemáticamente, este principio se escribe como:

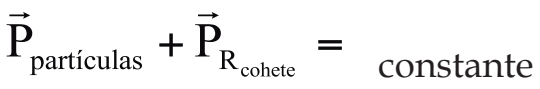

$\mathrm{O}$ también si $\mathrm{t}=\mathrm{t}_{0} \mathrm{y} \mathrm{t}=\mathrm{t}_{1}$ son dos instantes diferentes, este principio se escribe como:

$\left\{\overrightarrow{\mathrm{P}}_{\text {partículas }}+\overrightarrow{\mathrm{P}}_{\mathrm{R}_{\text {cohete }}}\right\}_{\mathrm{t}=\mathrm{t}_{0}}=\left\{\overrightarrow{\mathrm{P}}_{\text {particulas }}+\overrightarrow{\mathrm{P}}_{\mathrm{R}_{\text {cohete }}}\right\}_{\mathrm{t}=\mathrm{t}_{1}}$

Consideremos el instante $\mathrm{t}=\mathrm{t}_{0}$, como cualquier instante en el que el cohete estaba todavía en reposo en su plataforma de lanzamiento, entonces,

$$
\left\{\overrightarrow{\mathrm{P}}_{\text {partículas }}+\overrightarrow{\mathrm{P}}_{\mathrm{R}_{\text {cohete }}}\right\}_{\mathrm{t}=\mathrm{t}_{0}}=\{\overrightarrow{0}+\overrightarrow{0}\}=\overrightarrow{0}
$$

Por lo tanto, del principio de conservación de $\overrightarrow{\mathrm{p}}$ en cualquier otro instante $\mathrm{t}=\mathrm{t}_{1}$ en que el cohete ya está en movimiento, la cantidad de movimiento lineal debe conservarse, luego será igual al del instante $\mathrm{t}=\mathrm{t}_{0}$. Por lo anterior, podemos escribir que,

$$
\left\{\overrightarrow{\mathrm{P}}_{\text {partículas }}+\overrightarrow{\mathrm{P}}_{\mathrm{R}_{\text {cohete }}}\right\}_{\mathrm{t}=\mathrm{t}_{1}}=\overrightarrow{0}
$$


De esta última ecuación sabemos que en cualquier instante en el que el cohete se esté moviendo, se cumple que:

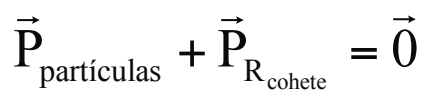

De donde, despejando $\overrightarrow{\mathrm{p}}_{\mathrm{R}_{\text {cohete }}}$ se obtiene que, $\overrightarrow{\mathrm{P}}_{\text {partículas }}=-\overrightarrow{\mathrm{P}}_{\mathrm{R}_{\text {cohete }}}$

O lo que es lo mismo, $\mathrm{m}_{\text {partículas }} \overrightarrow{\mathrm{v}}_{\text {partículas }}=-\mathrm{M}_{\mathrm{R}_{\text {cohete }}} \overrightarrow{\mathrm{V}}_{\mathrm{R}_{\text {cohte }}}$

Pero como la masa siempre es positiva, entonces, el signo negativo en la ecuación anterior nos indica que la dirección de la velocidad $\mathrm{V}_{\text {partículas }}$ de las partículas expulsadas de la tobera del cohete, es contraria a la dirección de la velocidad $\overrightarrow{\mathrm{V}}_{\mathrm{R}_{\text {cohete }}}$ del resto del cohete.

Entonces, si las partículas expulsadas por la tobera del cohete se mueven en una cierta dirección, el resto del cohete se moverá en una dirección contraria a la de las partículas. Así, en el momento del despegue del cohete, las partículas expulsadas se mueven verticalmente hacia abajo, lo que hace que el cohete se mueva verticalmente hacia arriba, y si el cohete está horizontal, y si los gases se mueven horizontalmente hacia la izquierda, el resto del cohete de moverá hacia la derecha.

Para calcular el momento lineal $\overrightarrow{\mathrm{P}}_{\text {particulas }}$ de los millones de partículas expulsadas por el cohete durante la combustión, hay que sumar los momentos lineales individuales de cada una de estas partículas, que a pesar de tener una masa muy pequeña, su momento lineal es muy considerable debido a sus altas velocidades, luego:

$\overrightarrow{\mathrm{P}}_{\text {particulas }}=\mathrm{m}_{1} \overrightarrow{\mathrm{v}}_{1}+\mathrm{m}_{2} \overrightarrow{\mathrm{v}}_{2}+\mathrm{m}_{3} \overrightarrow{\mathrm{v}}_{3}+\ldots+\mathrm{m}_{\mathrm{n}} \overrightarrow{\mathrm{v}}_{\mathrm{n}}$

Este momento lineal del conjunto de partículas expulsadas por la tobera permanece constante mientras dure la combustión que por esta se realiza utilizando siempre la misma proporción de combustible y oxidante.

Por otra parte, llamando $M_{R_{\text {cohece }}}$ a la masa del resto del cohete y llamando $\vec{V}_{R_{\text {conete }}}$ a la velocidad de éste, el momento lineal del resto del cohete $\mathrm{P}_{\mathrm{R}_{\text {cohete }}}$ está dado por: $\overrightarrow{\mathrm{P}}_{\mathrm{R}_{\text {cohete }}}=\mathrm{M}_{\mathrm{R}_{\text {cohete }}} \overrightarrow{\mathrm{V}}_{\mathrm{R}_{\text {cohete }}}$

Sin embargo, hay que notar que la masa $\mathrm{M}_{\mathrm{R}_{\text {conhec }}}$ del resto del cohete no es constante, puesto que al transcurrir el tiempo tanto la masa del combustible como 
la del oxidante disminuyen rápidamente al realizarse la combustión en la cámara del mismo nombre y por lo tanto, la masa del resto del cohete irá disminuyendo en la misma razón que disminuyen las masas del combustible y del oxidante.

Esto nos conduce a concluir que mientras se esté realizando la combustión, el oxidante y el combustible se irán consumiendo en una razón dada, es decir, cierto número de kilogramos por segundo, por lo tanto el empuje del cohete se mantiene constante mientras que la masa $\mathrm{M}_{\mathrm{R}_{\text {cohete }}}$ del resto $\mathrm{M}$ del cohete ira disminuyendo a razón del mismo número de kilogramos por segundo en que se quema la mezcla combustible-oxidante, pero como $\overrightarrow{\mathrm{P}}_{\mathrm{R}_{\text {cohete }}}=\mathrm{M}_{\mathrm{R}_{\text {cohete }}} \overrightarrow{\mathrm{V}}_{\mathrm{R}_{\text {cohete }}}$ debe mantenerse constante, entonces si la masa $\mathrm{M}_{\mathrm{R}_{\text {cohete }}}$ del cohete disminuye, el módulo $\mathrm{V}_{\mathrm{R}_{\text {cohete }}}$ de la velocidad $\mathrm{V}_{\mathrm{R}_{\text {cohete }}}$ del cohete tendrá que ir incrementándose para que el producto se mantenga constante.

Esa es la razón por la que una vez lanzado el cohete, su velocidad se incrementa en cada instante, hasta que se agoten el combustible y el oxidante, y la combustión finalice. Esta realidad, permite que el cohete no se queme por fricción con el aire al ir subiendo, ya que cercano a la superficie de la Tierra el aire es muy denso y la fricción en el cohete sería muy alta si su velocidad fuera muy grande, pero sucede lo contrario, el cohete inicia su movimiento desde un estado de reposo, y cuando su velocidad es muy grande a causa de la disminución de la masa del cohete, éste ya va muy alto y en esa región la densidad del aire es muy baja y la fricción sobre el cohete será prácticamente despreciable, llegando prácticamente a cero cuando ya no exista la atmósfera.

Alguien podría contradecir esto último alegando que la atmósfera de la Tierra se encuentra desde la superficie del planeta hasta una altura entre 1,000 y 1,300 $\mathrm{km}$, aproximadamente, si este es el caso, nuestra respuesta sería: lo que dices es cierto, pero hay que recordar que la mayoría de los gases que constituyen la atmósfera se encuentran en la zona cercana a la superficie terrestre, así, de acuerdo con la altura tenemos los siguientes valores:

El 50\% de la masa atmosférica está dentro de los primeros $5 \mathrm{~km}$.

El 75\% de la masa atmosférica está dentro de los primeros $11 \mathrm{~km}$.

E1 99.99\% de la masa atmosférica está dentro de los primeros $30 \mathrm{~km}$.

El $0.01 \%$ de la masa atmosférica se encuentra entre 30 y 1,300 km.

Por lo tanto, una vez que el cohete rebasa los $30 \mathrm{~km}$ de altura, la fricción con la atmósfera se puede despreciar sin pérdida de rigurosidad en el tratamiento del vuelo del cohete.

Tal vez algún lector tenga la inquietud de conocer ¿por qué un avión jet comercial o militar no puede llegar tan alto como un cohete?

La respuesta a esta interrogante es fácil (véase la figura 6), para que un avión jet vuele, requiere aire de donde extraer el oxígeno para efectuar la combustión en sus motores. Por eso los jets comerciales no vuelan a alturas muy altas $(10$ a 11 km como máximo) pues a partir de allí, el aire es de muy baja densidad y por ello el oxígeno es muy poco. 


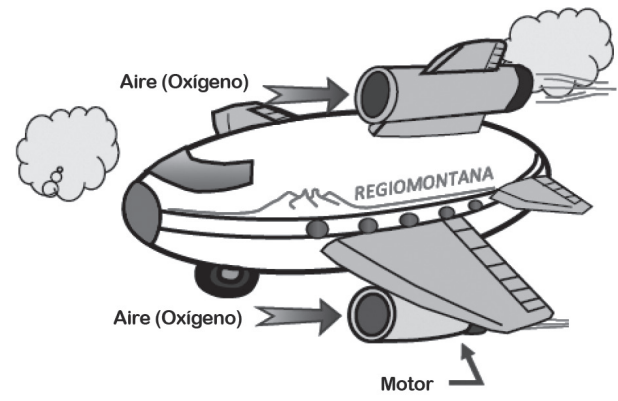

Figura 6. Un avión jet en pleno vuelo.

Para aclarar el porqué los aviones jet no llegan tan alto, veamos el funcionamiento de los motores de un avión jet (véase la Figura 7).

En la entrada de cada motor hay una especie de abanicos que succionan y comprimen el aire frío del medio ambiente, una vez comprimido el aire pasa a la cámara de combustión y se mezcla con el combustible, esta mezcla al incendiarse produce gases de alta presión y temperatura que mueven una turbina y ésta a su vez hace que se muevan los abanicos que succionan y comprimen el aire (véase la Figura 6).

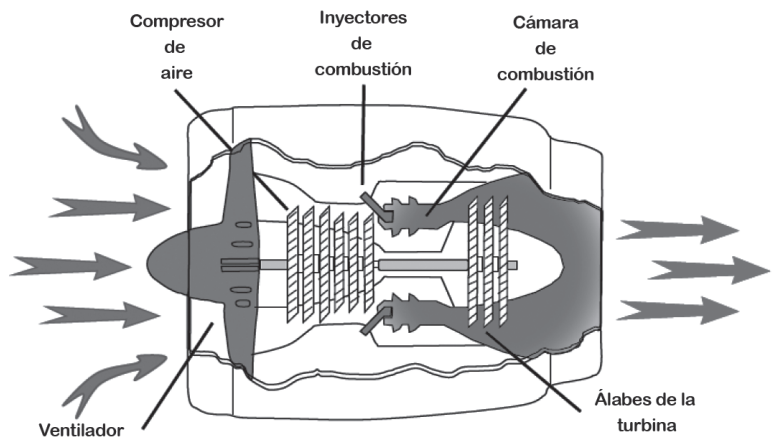

Figura 7. Motor de un avión jet.

El record mundial de altitud en un avión jet militar lo tiene el avión ruso Mikoyan-Gurevich MiG 25 lo estableció en 1977 y es de 37,500 m de altitud. Sin embargo, al llegar a esa altura, los motores se apagaron y el avión comenzó a caer libremente hasta que lo motores volvieron a funcionar. Este avión es capaz de alcanzar una velocidad de $3,500 \mathrm{~km} / \mathrm{h}$.

Por otra parte, el cohete de combustible líquido transporta su tanque de combustible y su tanque de oxidante (oxígeno). Por esa razón el cohete puede internarse en zonas donde no hay oxígeno, y llegar a otros planetas, y más allá del sistema planetario (véase la Figura 8). 